\title{
From the Editors' Desk: Decisions, Decisions
}

\author{
Mitchell D. Feldman, MD, MPhill and Richard L. Kravitz, MD, MSPH ${ }^{2}$ \\ 'Division of General Internal Medicine, University of California, San Francisco, San Francisco, CA, USA; ${ }^{2}$ University of California, Davis, \\ Sacramento, CA, USA.
}

J Gen Intern Med 26(4):353

DOI: $10.1007 / \mathrm{s} 11606-011-1649-1$

(C) The Author(s) 2011. This article is published with open access at Springerlink.com

I n his book, How Doctors Think, Jerome Groopman argues that medicine is an "uncertain science," and as such, medical decision-making often relies as much on intuition and experience as it does on evidence. In fact, Groopman cautions against the over-reliance on evidence-based decisionmaking, arguing that it must be balanced with the physician's own personal experience with the patient or the treatment. He contends that doctors "desperately need patients and their families and friends to help them think," and without this help, doctors are unlikely to make the correct diagnosis or give the most useful advice.

In this issue of JGIM, several articles examine the art and science of clinical decision-making. One way that doctors can use evidence to inform decision-making is by applying probability theory (or Bayes' theorem) to clinical decisions. While not eliminating uncertainty, it can, as suggested by Sox et al., "reduce uncertainty about uncertainty."

In this issue, Agoritsas et al. investigate the extent to which practicing doctors in Geneva, Switzerland, use disease prevalence when interpreting a positive test result. Prevalence matters, of course, in determining the post-test probability of disease (think HIV testing in a nunnery), but the study by Agoritsas et al. found that most physicians were unable to apply this concept when presented with a simple clinical scenario. This may lead to both unnecessary testing and diagnostic errors.

Few clinical decisions are as important as the determination of code status in a hospitalized patient. Anderson et al. examine audiotape code status discussions between attending hospitalist physicians and medical patients to better understand how these decisions are made. They found that despite the growing focus on this issue over the past decade, code status still is not discussed with many seriously ill patients, and when it is, the discussions are often incomplete. As Hauer and colleagues highlight in their research, part of the problem may reside in our not knowing how to adequately assess medical students' competence in shared decision-making. Key clinical decisions such as code status rely on shared decision-making between the doctor and patient. We must improve our ability to teach and assess this core skill in future physicians.

Published online February 17, 2011
Meanwhile, clinical investigators (including many JGIM readers) are engaged in a different sort of decision-making: where to submit the results of completed research. At JGIM, we're concerned about demands on authors' time and have taken steps to ease the submission process. Instructions for authors (http://www.jgimed.org/) are brief and (we hope) unambiguous. In contrast to many other journals, authors are requested to submit their manuscripts as a single Word document, inclusive of figures and tables. There is no cumbersome data entry process. And, on initial submission, we will accept references in any numbered format-no need to spend additional time finding the right EndNote reference style.

Another way we save authors' time is by promptly returning manuscripts thought unlikely to weather the editorial process. JGIM currently returns approximately $40 \%$ of original research submissions without external peer review. We do not do so lightly. In most cases, a co-Editor and/or Associate Editor and at least one other member of the editorial team review each manuscript. The most common reason for "reject without review" is lack of fit with the journal's scope, although we sometimes reject for lack of topicality, overlap with other recently published content, or major problems with clarity. We believe that prompt rejection gives authors an opportunity to submit their work to a more suitable venue without wasting their time.

Despite efforts at process improvement here and at other journals, the road from study completion to publication is often slow and precarious. One reason is that authors whose work is not accepted at one journal have to start the process all over again at the next. We are pleased to announce a new partnership between JGIM and the Annals of Internal Medicine that eliminates some of the redundancies. The Annals and JGIM have negotiated an arrangement such that authors who submit manuscripts to Annals can request at the time of submission that Annals send the manuscript directly to JGIM if after external review Annals decides not to publish it. This will greatly decrease the hassle factor for authors in resubmitting to JGIM after review at Annals and will allow JGIM to streamline our decision-making process for the submission. We are excited about this new venture and welcome your comments on this and other ways we can make JGIM more responsive to your needs.

Open Access: This article is distributed under the terms of the Creative Commons Attribution Noncommercial License which permits any noncommercial use, distribution, and reproduction in any medium, provided the original author(s) and source are credited.

Corresponding Author: Mitchell D. Feldman, MD, MPhil; Division of General Internal Medicine, University of California, San Francisco, 400 Parnassus Avenue, San Francisco, CA 94143-0320, USA (e-mail: Mitchell.Feldman@UCSF.edu). 